\section{Additional lesions seen in magnetic resonance imaging of breast cancer patients: the role of second-look ultrasound and imaging-guided interventions}

\author{
So Yoon Park ${ }^{1}$, Boo-Kyung Han ${ }^{1}$, Eun Sook Ko', Eun Young Ko ${ }^{1}$, Eun Yoon $\mathrm{Cho}^{2}$ \\ ${ }^{1}$ Department of Radiology and Center for Imaging Science, ${ }^{2}$ Department of Pathology, \\ Samsung Medical Center, Sungkyunkwan University School of Medicine, Seoul, Korea
}

Purpose: The purpose of this study was to investigate the final outcomes of magnetic resonance imaging (MRI)-identified additional lesions (MRALs) in breast cancer patients and the role of second-look ultrasound (SLUS) and imaging-guided interventions.

Methods: We analyzed breast cancer patients with MRALs on preoperative MRI between January and June 2012. MRALs were defined as additional lesions suspected on MRI but not suspected on mammograms or ultrasound. The malignancy rate of MRALS, MRI-based Breast Imaging Reporting and Database System (BI-RADS) category, positional relationship with the index cancer, MRI-concordant lesion visibility on SLUS, performance of imaging-guided interventions, and total mastectomy (TM) rates were evaluated for the confirmed lesions.

Results: Among the 119 confirmed lesions, SLUS and imaging-guided interventions were performed in $94(79.0 \%)$ and 82 cases (68.9\%), respectively. The malignancy rate was $68.1 \%$ (81 of 119), and was significantly higher in BI-RADS 4C-5 lesions than in 4A-4B lesions $(94.6 \%$ vs. $56.1 \%, P<0.01)$ and in ipsilateral same-quadrant lesions than in contralateral lesions $(84.2 \%$ vs. $33.3 \%, P<0.01)$. The lesion visibility rate on SLUS was $90.4 \%$. The malignancy rate was not significantly different according to lesion visibility on SLUS. The TM rate in the 98 cases with ipsilateral MRALs was $37.8 \%$, while it was significantly lower in patients who underwent an imaging-guided intervention than in those who did not (27.9\% vs. $54.1 \%, \mathrm{P}=0.017)$.

Conclusion: MRALs show a high probability of malignancy, especially if they are ipsilateral. SLUS and imaging-guided interventions can eliminate many unnecessary TMs.

Keywords: Breast neoplasms; Magnetic resonance imaging; Ultrasonography

\section{Introduction}

Breast magnetic resonance imaging (MRI) is an important imaging modality for the diagnosis of breast cancer because of its high sensitivity of $68 \%-100 \%$ [1-3]. In patients with cancer, MRI is known to reveal additional cancers that are occult on mammography or ultrasonography (US) in a median of $16 \%$ of patients [1-5]. However, because of its relatively low specificity (range, $37 \%$ to
ULTRA
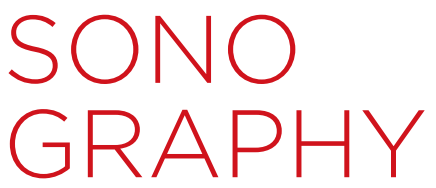

ORIGINAL ARTICLE

https://doi.org/10.14366/usg. 18002 pISSN: 2288-5919 • elSSN: 2288-5943

Ultrasonography 2019;38:76-82

Received: January 8, 2018

Revised: June 21, 2018

Accepted: June 23, 2018

Correspondence to:

Boo-Kyung Han, MD, Department of Radiology and Center for Imaging Science, Samsung Medical Center, Sungkyunkwan University School of Medicine, 81 Irwon-ro, Gangnam-gu, Seoul 06351, Korea

Tel. $+82-2-3410-2518$

Fax. +82-2-3410-2559

E-mail: bkhan@skku.edu

This is an Open Access article distributed under the terms of the Creative Commons Attribution NonCommercial License (http://creativecommons.org/ licenses/by-nc/3.0/) which permits unrestricted noncommercial use, distribution, and reproduction in any medium, provided the original work is properly cited.

Copyright (C) 2019 Korean Society of Ultrasound in Medicine (KSUM)

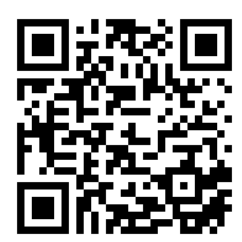

How to cite this article:

Park SY, Han BK, Ko ES, Ko EY, Cho EY. Additional lesions seen in magnetic resonance imaging of breast cancer patients: the role of second-look ultrasound and imaging-guided interventions. Ultrasonography. 2019 Jan;38(1):76-82. 
97\%), MRI may increase unnecessary total mastectomy (TM) or wider excision, and false-positive lesions can increase patient anxiety [6-10]. Furthermore, no study has shown that MRI reduced the reoperation rates. A few studies suggested that even the additional lesions detected by MRI can be controlled by nonsurgical treatments, including radiation and systemic therapy $[3,11,12]$. Nonetheless, MRI is increasingly used to reduce the amount of residual disease detected during postoperative surveillance $[3,10,13]$.

Many studies have emphasized the need to obtain biopsies of all lesions, and therefore, the role of second-look ultrasonography (SLUS) for MRI-identified additional lesions (MRALs) has been highlighted $[4,6,14-16]$. SLUS facilitates the detection of MRI-concordant lesions, provides morphological and vascular information, and helps predict the pathology of the lesions. SLUS is well-tolerated, cost-effective, and time-saving for patients and surgeons awaiting an urgent operation. However, limited data are available on the validation of these lesions after SLUS in a clinical setting in terms of the value and frequency of imaging-guided interventions. The goal of our study was to investigate suspected MRALs in breast cancer patients and to characterize the role of SLUS and imaging-guided interventions.

\section{Materials and Methods}

The study was approved by the institutional review board of Samsung Medical Center and the requirement for informed consent from the patients was waived.

\section{Patients}

A retrospective review of the MRI database at our institution identified 695 consecutive breast cancer patients who underwent preoperative MRI from January to June 2012, with surgical management within a month. Excluding 39 patients who underwent neoadjuvant chemotherapy before surgery and 72 patients who were diagnosed with multicentric or bilateral cancers before MRI, 584 patients who were originally diagnosed with a unifocal cancer were identified (mean age, 50 \pm 17.6 years; range, 30 to 81 years). Among them, we searched for women who had at least one additional suspicious lesion that was occult on initial mammography and ultrasound, but was recommended for additional histological confirmation after MRI.

\section{Breast MRI}

Breast MRI was performed using an Intera Achieva 3.0-T or 1.5-T apparatus (both from Philips Medical Systems, Best, The Netherlands) with dedicated breast coils. Patients were placed in the prone position. The MRI protocol included axial T2-weighted and dynamic T1-weighted fat-saturated sequences obtained in pre-contrast and post-contrast $(60,120,180,240,300$, and 360 seconds after contrast administration) phases for bilateral breasts. Gadobutrol (Gadovist, Bayer Healthcare, Berlin, Germany) was intravenously injected according to the patient's weight using the formula of $0.1 \mathrm{mmol} / \mathrm{kg}$.

Image Interpretation, SLUS, and Imaging-Guided Interventions MRI images were interpreted by one of four board-certified radiologists specializing in breast imaging, and all were routinely compared with prior mammograms and US. If abnormal lesions other than the index cancers were identified and required additional histological confirmation, even though they were occult or benignseeming on prior images, we considered them to be suspicious and classified them according to the American College of Radiology Breast Imaging Reporting and Data System (BI-RADS) into categories $4 \mathrm{~A}, 4 \mathrm{~B}, 4 \mathrm{C}$, or 5 . If the radiologist thought the suspicious lesion might potentially be visualized by US, SLUS was recommended. If microcalcifications were detected during the reassessment of mammography, mammography-guided localization surgery was recommended. If the radiologist who read the MRI considered neither option to be feasible or if the radiologist who performed SLUS considered a lesion still suspicious even after negative SLUS results, MRI-guided biopsy was recommended. SLUS was performed by one of six breast radiologists with 5 to 17 years of experience in breast US and MRI. The scanning was focused on the area with the MRALS, and the lesions on US were deemed to be concordant with the MRALs when the location, depth, size, and shape of the lesions were compatible with the MRI findings [5]. If the radiologists deemed that the MRI-concordant lesions were clearly visible on US, US-guided core biopsy was performed. If the radiologist deemed that areas of non-mass enhancement or foci equal or smaller than $5 \mathrm{~mm}$ were not amenable to core biopsy, the other option was US-guided localization surgery. A total of 157 cases showed additional lesions that were classified as BI-RADS category 4 or 5 . A breast imaging radiologist (B.K.H., with 17 years of experience in breast imaging) reviewed the $157 \mathrm{MRI}$ images and determined their eligibility under the original criteria for MRALs, considering the distance from the index cancer. The criteria for MRALs were contralateral breast lesions, ipsilateral lesions more than $1 \mathrm{~cm}$ apart from the index cancers or located in a different quadrant, or ipsilateral non-mass lesions contiguous with the index tumor that extended more than 2 $\mathrm{cm}$ beyond the presumed site of the index cancer. These criteria were modified from a previous study [17] to facilitate better aesthetic outcomes of conservative breast surgery.

US-guided biopsy was performed with a 14-G Tru-cut automated biopsy gun (ACECUT, TSK Laboratory, Tochigi, Japan) or an 11-G 
vacuum-assisted device (Mammotome, Devicor, Cincinnati, OH, USA). Wire localization entailed the insertion of hook wires (Accura BLN, Argon Medical Device, Athens, TX, USA) under US or mammography guidance. US-guided tattooing was performed using a 3\% charcoal suspension ( $0.3 \mathrm{~g}$ of activated charcoal in $10 \mathrm{~mL}$ saline) prepared at the pharmacy department of our institution and injected into the lesions through a needle tract antiparallel to the US probe. MRIguided biopsy was performed using an ATEC 9-G vacuum-assisted device (Hologic, Marlborough, MA, USA).

\section{Pathology Results and Follow-up}

The final results of MRALs were divided into benign and malignant. Benign MRALs include benign or borderline lesions (atypia and radial scars), which were confirmed by operative biopsy or imaging follow-up for more than 2 years via US or MRI findings that showed stability or disappearance. Seven cases underwent MRI followup and the rest were followed up with US. Malignant MRALS included pathologically confirmed invasive breast cancers or ductal carcinoma in situ (DCIS). If a patient had more than one MRAL, we selected the most suspicious lesion for analysis. MRALs that were surgically resected using a non-localization method were included only when surgeons performed wider excision based on MRI with an intraoperative frozen section or the radiologists directly communicated with the pathologist to determine whether a pathologically identified lesion matched the area corresponding to MRALs, and when the lesion was stable during follow-up in benign cases.

\section{Data Analysis}

The imaging data and pathology results of the patients and the type of surgery (TM vs. breast-conserving surgery [BCS]) were investigated in our hospital information system. We analyzed the malignancy rate of MRALs according to the BI-RADS category, the positional relationship with the index cancer, MRI-concordant lesion visibility on SLUS, and the imaging-guided intervention method for MRALs. Based on the positional relationship, the location of MRALs was classified as ipsilateral in the same quadrant, ipsilateral in a different quadrant, or contralateral. The TM rate in cases of ipsilateral MRALs was compared according to each variable.

\section{Statistical Analyses}

Statistical analyses were performed using SPSS version 19 (IBM Corp., Armonk, NY, USA). The malignancy rate of all MRALs and the TM rate for ipsilateral MRALs were analyzed according to their BIRADS category, positional relationship with the index cancer, MRIconcordant lesion visibility on SLUS, and whether an imaging-guided intervention was performed, using the chi-square test or the Fisher exact test.

\section{Results}

\section{MRI, SLUS, and Imaging-Guided Interventions}

Among 584 cases of preoperative MRI, 157 (26.9\%) showed additional lesions classified as BI-RADS category 4 or 5 . Excluding the 20 cases that failed to meet the distance criteria and 18 cases without a detailed description of the pathological outcome for the MRALs and imaging follow-up data for that site, 119 cases (20.3\%) showed MRALs within the reference standard. The median size of the MRALs on MRI was $1.0 \mathrm{~cm}$ (range, 0.3 to $7.0 \mathrm{~cm}$ ). The morphological type of the MRALs was a mass in 56 cases, a nonmass-like enhancement in 53, and a focus in 10.

Fig. 1 shows a flow chart of the imaging and intervention workup of the 119 cases with MRALs. SLUS was performed in 94 cases $(79.0 \%)$, and $85(90.4 \%)$ of these showed MRI-concordant lesions. Of these cases, 77 were confirmed by a US-guided biopsy and the decision between BCS and TM followed the pathologic results, while eight lesions were excluded from US-guided biopsy at the radiologist's discretion in the absence of the potential to be benign based on the combined assessment of MRI and US. Among the remaining nine cases of MRALs not detected on SLUS, two underwent MRI-guided biopsy, six were treated with additional surgery with a non-localization wider-excision method, and one did not undergo surgical biopsy and was left untouched. MRI followup revealed disappearance of the lesions. Of the 25 cases without SLUS, three were localized under mammography guidance.

Imaging-guided core or localization biopsies were performed in 82 cases $(68.9 \%)$ via US, MRI, or mammography guidance in 77 , two, and three cases, respectively. Among the 37 cases (31.1\%) without imaging-guided lesion localization, surgeons performed an intraoperative frozen-section biopsy based on MRI findings in 36 cases. Mammography-guided localization biopsy was performed for lesions that were later identified via a secondary reading after MRImammography correlation.

During the study period, 22 cases of MRALs were directly treated with surgery without SLUS, preoperative biopsy, or localization. These patients included 13 cases in the MRI-based BI-RADS 4C or 5 category, and additional surgery, including TM, was indicated due to incremental malignant lesions. The remaining nine cases with lesions classified as BI-RADS 4A or 4B based on MRI ( $11 \%$ of these 2 categories) skipped the confirmation procedure due to a tight schedule between MRI and surgery. Additional surgery was justified in six cases due to incremental malignant lesions and unjustified in three cases due to the benign nature of the lesions. 


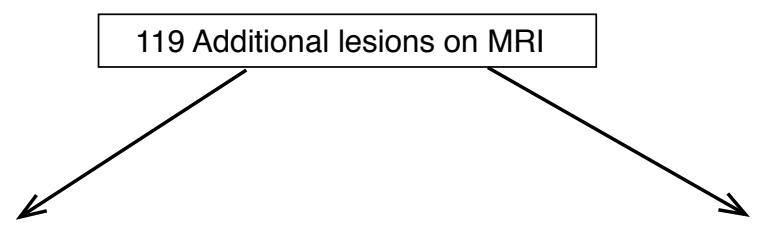

\section{Second-look US}

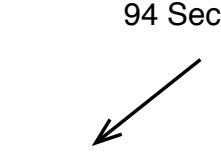

85 Visible
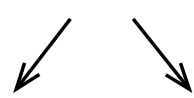

Imaging-guided intervention

\section{US-guided 8 No}

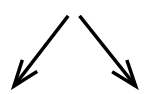

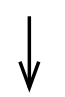

Surgery

28 Benign 49 Malignant 8 Malignant
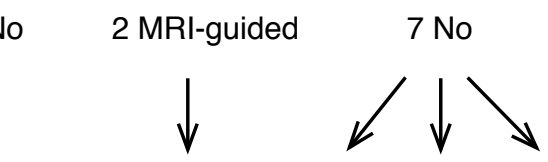

9 Occult

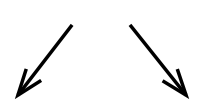

25 Second-look US (-)

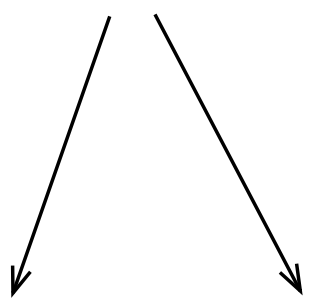

3 Mammography-guided ${ }^{\text {a) }}$
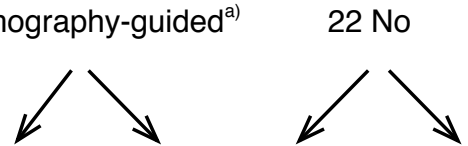

1 Untouched

2 Benign 1 Malignant 3 Benign 19 Malignant

Fig. 1. Work-up for MRI-identified additional lesions. MRI, magnetic resonance imaging; US, ultrasound; FU, follow-up. ${ }^{\text {a) } C a l c i f i c a t i o n s ~ w e r e ~}$ retrospectively detected on mammography after MRI revealed abnormal findings.

Table 1. Final outcomes of MRALs $(\mathrm{n}=119)$

\begin{tabular}{lccc}
\hline & $\begin{array}{c}\text { No. of } \\
\text { cases (\%) }\end{array}$ & $\begin{array}{c}\text { Malignancy } \\
\text { rate (\%) }\end{array}$ & P-value \\
\hline MRI BI-RADS category & & & \\
4A & $48(40.3)$ & 54.2 & $<0.001$ (overall) \\
4B & $34(28.6)$ & 58.8 & 0.847 (4A vs. 4B) \\
4C & $21(17.6)$ & 90.5 & 0.015 (4B vs. 4C) \\
5 & $16(13.4)$ & 100 & 0.496 (4C vs. 5) \\
& & & \\
4A-4B & $82(68.9)$ & 56.1 & $<0.001$ \\
4C-5 & $37(31.1)$ & 94.6 & \\
Position of MRALs & & & \\
Ipsilateral same quadrant & $38(31.9)$ & 84.2 & $<0.001$ \\
Ipsilateral different quadrant & $60(50.4)$ & 70.0 & \\
Contralateral & $21(17.6)$ & 33.3 & \\
Second-look US & & & \\
MRI-concordant lesions (+) & $85(71.4)$ & 67.1 & 0.271 \\
MRI-concordant lesions (-) & $9(7.6)$ & 44.4 & \\
Not done & $25(21.0)$ & 80.0 & \\
Imaging-guided intervention & & & \\
Yes & $82(68.9)$ & 61.0 & \\
No & $37(31.1)$ & 83.8 & \\
Total & $119(100)$ & 68.1 & \\
\hline MRALs, MR-ident &
\end{tabular}

MRALS, MRI-identified suspicious additional lesions; MRI, magnetic resonance imaging; BI-RADS, Breast Imaging Reporting and Database System; US, ultrasound.

\section{Final Results and Surgery}

Among the 119 MRALs, 81 (68.1\%) were malignant. Table 1 shows the final outcome of the MRALs according to each variable. The malignancy rate of the MRALs differed significantly according to the MRI-based BI-RADS category $(\mathrm{P}<0.001)$. The rate was not significantly different between BI-RADS 4A (54.2\%) and 4B (58.8\%) lesions, but that of BI-RADS 4C-5 (94.6\%) lesions was significantly higher than that of BI-RADS 4A-4B lesions $(56.1 \%, P<0.001)$. The malignancy rate of MRALs was significantly different according to their position $(\mathrm{P}<0.001)$. It was the highest in ipsilateral samequadrant MRALs (84.2\%) and the lowest in MRALs located in the contralateral breast (33.3\%). The malignancy rate of MRALs was higher in cases with MRI-concordant lesions on SLUS than in those without (67.1\% vs. $44.4 \%$, respectively), but the difference was not statistically significant $(P=0.271)$. The malignancy rate was significantly higher $(\mathrm{P}=0.012)$ in MRALs for which an imagingguided intervention was not performed (83.8\%) than in MRALs for which imaging-guided intervention was performed (61.0\%). Among the 81 malignant lesions, 38 were DCIS and 43 were invasive cancers.

Table 2 presents the TM rates in patients with MRALs in the ipsilateral breast. Among the 98 patients diagnosed with ipsilateral MRALS, 37 (37.8\%) underwent TM. The TM rate differed significantly according to the BI-RADS category of the MRALs $(P=0.003)$; the rate was significantly higher in BI-RADS 4C-5 (61.1\%) lesions 
Table 2. Total mastectomy rate in breast cancer patients with ipsilateral MRALs $(n=98)$

\begin{tabular}{lcccc}
\hline & $\begin{array}{c}\text { No. of } \\
\text { cases } \\
(\%)\end{array}$ & $\begin{array}{c}\text { Total } \\
\text { mastectomy }\end{array}$ & $\begin{array}{c}\text { Breast- } \\
\text { conserving } \\
\text { surgery }\end{array}$ & P-value \\
\hline $\begin{array}{l}\text { MRI BI-RADS category } \\
\text { 4A }\end{array}$ & $35(35.7)$ & $9(25.7)$ & $26(74.3)$ & 0.003 \\
(overall)
\end{tabular}

Values are presented as number (\%).

MRALs, MRI-identified suspicious additional lesions; MRI, magnetic resonance imaging; BI-RADS, Breast Imaging Reporting and Database System.

than in BI-RADS 4A-4B (24.2\%) lesions. The TM rate was slightly, but not significantly, higher $(\mathrm{P}=0.152)$ in patients with ipsilateral different-quadrant lesions (43.3\%) than in those with ipsilateral same-quadrant lesions (28.9\%). Of the 61 patients who underwent imaging-guided core or localization biopsy, TM was performed in 17 (27.9\%), and the TM rate was significantly lower than in patients who did not undergo an imaging-guided intervention (20 of 37, $54.1 \%)(P=0.017)$. Twenty-seven of the 38 patients $(71.1 \%)$ with ipsilateral same-quadrant MRALs were treated with BCS, with the remaining 11 were treated with TM because the additional lesion was larger than $4 \mathrm{~cm}$. Among the 60 cases in which MRALs were detected in a different quadrant, 27 (45.0\%) avoided TM through undergoing an imaging-guided intervention.

\section{Discussion}

MRI shows a higher sensitivity for breast cancers than either mammography or US, and the applications and techniques of MRI has been improved significantly in recent years $[1,3]$. Researchers arguing against preoperative breast MRI have suggested that it increases the detection of additional lesions and contributes to a higher TM rate without providing a definite survival benefit $[17,18]$.
These findings are mainly based on the analysis of clinical outcomes, including re-excision rates, conversion to mastectomy, local recurrence, and contralateral cancer [2]. Few studies have analyzed pathological outcomes based on a per-lesion analysis because it is difficult to examine all radiographically detected diseases. We investigated the pathological outcomes of MRALs by validating the most suspicious lesions using all possible methods, including SLUS and imaging-guided interventions. The malignancy rate of MRALs was $68.1 \%$, and the remaining cases were benign, suggesting that SLUS and imaging-guided intervention were appropriate for the evaluation of MRALs. The malignancy rate of MRALs reached $94.6 \%$ in the BI-RADS 4C-5 category. Considering the high possibility of malignancy, if a lesion is assessed as BI-RADS 4C-5, surgery should be planned for the removal of these lesions after pathologic confirmation using preoperative localization or biopsy. The falsepositive rate of $5.4 \%$ should be considered. The malignancy rate of BI-RADS 4 A lesions was $54.2 \%$, which was much higher than the rate of lesions discovered by screening mammography or US. Therefore, MRALS should be managed more carefully than screening-detected lesions and should be confirmed by SLUS or pathological confirmation by other means. However, wider surgery or TM without pathological verification is not desirable because 31.9\% of all MRALs were benign. Whereas $75.5 \%$ of ipsilateral MRALs (74 of 98) were malignant, only $33.3 \%$ of contralateral MRALs (7 of 21) were proven malignant. Thus, contralateral MRALs showed a higher false-positive rate than ipsilateral MRALs.

In our study, SLUS was performed in $79.0 \%$ of MRALs (94 of 119) and $90.4 \%$ of them (85 of 94 ) showed MRI-concordant lesions. The detection rate was somewhat higher than previously reported rates ranging from $46 \%$ to $86.8 \%$ [4-6]. Our radiologists are very committed to identifying MRI-concordant lesions by focusing on the anatomic information and lesion-associated hypervascularity, and they had experienced a large volume of clinical cases. A weekly perlesion pathological analysis was one of the key steps in enhancing the outcomes of SLUS. The non-visualization rate of malignant lesions on SLUS was $6.6 \%$ (4 of 61).

In our study, the incidence of MRALs was 20.3\% (119 of 584) except for unvalidated cases and clinically insignificant cases. The reported rates of detection of additional lesions by MRI vary between $6 \%$ and $27 \%[3,19]$, in accordance with our study. According to previous reports that analyzed pathologic outcomes, the rate of multifocal (1 quadrant involved) or multicentric (multiple quadrants involved) cancer ranged between $14 \%$ and $47 \%[17,20]$. We found a rate of $12.7 \%$ (74 of 584 ). However, our study was not about all cases of multifocal or multicentric lesions; instead, it investigated clinically and conventional imaging-based occult 
multifocal or multicentric lesions that were only identified by MRI.

We performed imaging-guided interventions in 43 of 60 ipsilateral different-quadrant cases, which are often treated with TM. With the help of our study procedure, 13 cases with verified benign lesions and 14 cases with verified malignant lesions avoided TM. The TM rate in the 98 cases with ipsilateral MRALs was $37.8 \%$, which was significantly lower in patients who underwent imagingguided interventions than in those who did not (27.9\% vs. $54.1 \%$, $\mathrm{P}=0.012$ ). Breast conservation and eradication of malignant foci are feasible through imaging-guided localization and multiquadrant surgery, suggesting that radiological and surgical interventions are ideal. What is essential is that both surgeons and radiologists should accept the value of these efforts.

There are some limitations of our study. First, this study was a retrospective analysis of patients treated at a single institution based on radiological imaging and pathological reports. Second, in 28 patients treated with surgery using a non-localization widerexcision method, surgical description and communication between radiologists and pathologists were the only tools to demonstrate the lesion concordance and its outcome, suggesting that this analysis may have been subjective. Third, US-guided core or localization biopsies were much more frequent than MRI-guided biopsies. Despite the convenient and cost-effective nature of SLUS, using US or mammography to guide the treatment of MRI-identified lesions always encounters the issue of lesion matching. However, we believe that MRI or US follow-up could confirm lesion concordance. Recent trends suggest that it can be useful to insert marker clips following US-guided biopsies.

In conclusion, MRALs in breast cancer patients showed a high probability of malignancy. SLUS and imaging-guided interventions prevented needless wide-extent surgery due to false-positive MRI findings. MRI-related poor aesthetic outcomes were also avoided.

ORCID: So Yoon Park: https://orcid.org/0000-0001-9254-0220; Boo-Kyung Han: https://orcid.org/0000-0003-1896-0571; Eun Sook Ko: https://orcid.org/0000-00020399-7956; Eun Young Ko: https://orcid.org/0000-0001-6679-9650; Eun Yoon Cho: https://orcid.org/0000-0003-4675-4492

\section{Conflict of Interest}

No potential conflict of interest relevant to this article was reported.

\section{References}

1. Berg WA, Zhang Z, Lehrer $D$, Jong RA, Pisano ED, Barr RG, et al. Detection of breast cancer with addition of annual screening ultrasound or a single screening MRI to mammography in women with elevated breast cancer risk. JAMA 2012;307:1394-1404.
2. Morrow M, Waters J, Morris E. MRI for breast cancer screening, diagnosis, and treatment. Lancet 2011;378:1804-1811.

3. Fan XC, Nemoto T, Blatto K, Mangiafesto E, Sundberg J, Chen A, et al. Impact of presurgical breast magnetic resonance imaging (MRI) on surgical planning: a retrospective analysis from a private radiology group. Breast J 2013;19:134-141.

4. Hong MJ, Cha JH, Kim HH, Shin HJ, Chae EY, Shin JE, et al. Secondlook ultrasonography for MRI-detected suspicious breast lesions in patients with breast cancer. Ultrasonography 2015;34:125-132.

5. Lee $S H$, Kim SM, Jang M, Yun BL, Kang E, Kim SW, et al. Role of second-look ultrasound examinations for MR-detected lesions in patients with breast cancer. Ultraschall Med 2015;36:140-148.

6. Nam SJ, Kim EK, Kim MJ, Moon HJ, Yoon JH. Significance of incidentally detected subcentimeter enhancing lesions on preoperative breast MRI: role of second-look ultrasound in lesion detection and management. AJR Am J Roentgenol 2015;204:W357-W362.

7. Parsian $\mathrm{S}$, Rahbar $\mathrm{H}$, Allison $\mathrm{KH}$, Demartini WB, Olson ML, Lehman $C D$, et al. Nonmalignant breast lesions: ADCs of benign and highrisk subtypes assessed as false-positive at dynamic enhanced MR imaging. Radiology 2012;265:696-706.

8. Katipamula R, Degnim AC, Hoskin T, Boughey JC, Loprinzi C, Grant $\mathrm{CS}$, et al. Trends in mastectomy rates at the Mayo Clinic Rochester: effect of surgical year and preoperative magnetic resonance imaging. J Clin Oncol 2009;27:4082-4088.

9. Nijenhuis MV, Rutgers EJ. Conservative surgery for multifocal/ multicentric breast cancer. Breast 2015;24 Suppl 2:S96-S99.

10. Lai HW, Chen CJ, Lin YJ, Chen SL, Wu HK, Wu YT, et al. Does breast magnetic resonance imaging combined with conventional imaging modalities decrease the rates of surgical margin involvement and reoperation?: a case-control comparative analysis. Medicine (Baltimore) 2016;95:e3810.

11. Okumura S, Mitsumori M, Yamauchi C, Kawamura S, Oya N, Nagata $Y$, et al. Feasibility of breast-conserving therapy for macroscopically multiple ipsilateral breast cancer. Int J Radiat Oncol Biol Phys 2004;59:146-151.

12. Cho LC, Senzer N, Peters GN. Conservative surgery and radiation therapy for macroscopically multiple ipsilateral invasive breast cancers. Am J Surg 2002;183:650-654.

13. Houvenaeghel G, Tallet A, Jalaguier-Coudray A, Cohen M, Bannier $M$, Jauffret-Fara $C$, et al. Is breast conservative surgery a reasonable option in multifocal or multicentric tumors? World J Clin Oncol 2016;7:234-242.

14. Park VY, Kim MJ, Kim EK, Moon HJ. Second-look US: how to find breast lesions with a suspicious MR imaging appearance. Radiographics 2013;33:1361-1375.

15. Leung JW. Utility of second-look ultrasound in the evaluation of MRI-detected breast lesions. Semin Roentgenol 2011;46:260-274.

16. Spick C, Baltzer PA. Diagnostic utility of second-look US for breast 
lesions identified at MR imaging: systematic review and metaanalysis. Radiology 2014;273:401-409.

17. Liberman L, Morris EA, Dershaw DD, Abramson AF, Tan LK. MR imaging of the ipsilateral breast in women with percutaneously proven breast cancer. AJR Am J Roentgenol 2003;180:901-910.

18. Miller BT, Abbott AM, Tuttle TM. The influence of preoperative MRI on breast cancer treatment. Ann Surg Oncol 2012;19:536-540.

19. Schell AM, Rosenkranz K, Lewis PJ. Role of breast MRI in the preoperative evaluation of patients with newly diagnosed breast cancer. AJR Am J Roentgenol 2009;192:1438-1444.

20. Morrow M. Limiting breast surgery to the proper minimum. Breast 2005;14:523-526. 